\title{
Síndrome de la costilla deslizante. Tratamiento agresivo pero eficaz
}

\section{Slipping rib syndrome. An aggressive but effective treatment}

\author{
N. González Temprano, L. Ayuso González, S. Hernández Martín, \\ A.Y. Molina Caballero, J. Pisón Chacón, M.A. Martínez Bermejo
}

\section{RESUMEN}

El síndrome de la costilla deslizante (SCD) es una entidad infrecuente, que exige un diagnóstico diferencial preciso incluyendo patología digestiva, cardiaca, respiratoria, infecciosa y músculo-esquelética torácica o abdominal.

Se presenta el caso de dos niñas, en edad puberal, con dolor torácico incapacitante de varios meses de evolución y sin antecedente traumático. En la exploración existía un punto de dolor a la palpación de la parrilla costal y la "maniobra del gancho" era dolorosa. Se realizó una resección de los cartílagos costales, respetando el pericondrio. La evolución tras uno y cuatro años de seguimiento, fue satisfactoria sin complicaciones y con resolución inmediata de la sintomatología.

La cirugía de resección costal parece una opción muy agresiva, aunque con resultados muy satisfactorios. Un abordaje mínimamente invasivo podría ser mejor opción a considerar en el futuro.

Palabras clave. Dolor torácico recurrente. Costocondritis. Síndrome de Tietze. Pediatría. Síndrome doloroso costal inferior.

\begin{abstract}
Slipping rib syndrome (SRS) is an unusual cause of recurrent chest or abdominal pain in children. The diagnosis is elusive, including gastroenterological, cardiac, respiratory, infectious and chest or abdominal muscular pathologies.

Two paediatric patients were diagnosed with SRS, both of them were female teenagers with a similar clinical pattern: crippling unilateral chest pain without a traumatic event. On physical examination, all patients had reproducible pain with the "hooking maneuver". Surgical excision of the costal cartilages was done, preserving the perichondrium. No complications were reported. In both cases we achieve an excellent outcome after one and four years of follow-up, resolving the symptoms completely.

The surgical excision of the costal cartilages seems to be an aggressive option but with an excellent outcome. A minimum invasive approach could be a better option in the future.
\end{abstract}

Keywords. Recurrent chest pain. Costochondritis. Tietze syndrome. Paediatric. Slipping rib syndrome.
Servicio de Cirugía Pediátrica. Complejo Hospitalario de Navarra.

Recepción: 14 de agosto de 2014

Aceptación provisional: 17 de diciembre de 2014

Aceptación definitiva: 24 de febrero de 2015

\section{Correspondencia:}

Nerea González Temprano

Servicio de Cirugía Pediátrica

Complejo Hospitalario de Navarra

$\mathrm{C} \backslash$ Irunlarrea, 4

31008 Pamplona

E-mail: pika_netxu@yahoo.es 


\section{INTRODUCCIÓN}

El síndrome de costilla deslizante (SCD) se produce por una movilidad excesiva de los cartílagos costales inferiores por inadecuada fijación ligamentosa, produciendo una subluxación de los mismos y comprimiendo así los nervios intercostales contiguos. Este síndrome ha sido descrito en numerosos trabajos ${ }^{1-5}$. Al ser un síntoma sin signos acompañantes, es muy difícil concretar el diagnóstico. La historia clínica y la exploración son fundamentales, ya que el dolor en la región torácica plantea un amplio diagnóstico diferencial (Tabla 1). Se debe descartar desde patología torácica, incluyendo cardiaca, respiratoria y músculo-esquelética hasta patología abdominal, tanto gastrointestinal, renal o biliar.
El conocimiento y la difusión de esta infrecuente entidad podrían evitar multitud de pruebas diagnósticas, algunas de ellas agresivas, en estos enfermos.

En el presente trabajo se han revisado los pacientes pediátricos diagnosticados de SCD desde 2006 a 2014, aportando una revisión bibliográfica de esta entidad y una sugerencia terapéutica.

\section{CASOS CLÍNICOS}

\section{Caso 1}

Paciente de 13 años que presenta dolor torácico derecho incapacitante de 3 meses de evolución, que no calma con analgésicos. Sin antecedente traumático.

La palpación de los cartílagos costales es dolorosa, refiriendo como punto más doloroso

Tabla 1. Diagnóstico diferencial de dolor torácico en pediatría

\begin{tabular}{|l|l|}
\hline Idiopático & \\
\hline Músculo-esquelético & - Traumatismo \\
& - Sobrecarga muscular \\
& - Costocondritis \\
& - Síndrome de Tietze \\
& - Síndrome de pinzamiento precordial \\
\hline Respiratorio & - Asma de costilla deslizante \\
\hline Psicógeno & - Neumonía \\
& - Derrame pleural \\
\hline Gastrointestinal & - Neumotórax \\
& - Pleurodinia \\
\hline Cardiovascular & - Hiperventilación \\
& - Conversión \\
\hline & - Esoflujo gastroesofágico \\
& - Gastritis \\
& - Ingesta de cuerpo extraño/cáustico \\
& - Anomalías cardiacas estructurales \\
& - Fenómenos inflamatorios: miocarditis, pericarditis... \\
& - Enfermedad de Kawasaki \\
& - Vasoespasmo coronario \\
& - Tromboción aórtica \\
& - Mastalgia \\
& - Herpes zóster \\
\hline
\end{tabular}


la $7^{\mathrm{a}}$ unión costoesternal derecha, sin apreciarse alteraciones en el contorno ni signos inflamatorios cutáneos. La maniobra del gancho, que consiste en producir una elevación de los cartílagos costales inferiores mediante la introducción de los dedos del explorador por debajo del último arco costal y traccionando de él en sentido craneal, es muy dolorosa. El resto de exploración así como la radiografía de tórax es normal.

Dada la exploración tan característica, se pensó en el SCD y se optó por un tratamiento conservador con antiinflamatorios orales.

Tras un mes sin mejoría, se le propuso bloqueo anestésico intercostal o tratamiento quirúrgico definitivo. Dado que el dolor de la niña cada vez es más limitante y que la primera opción aportaría un alivio temporal, se realizó la intervención quirúrgica. $\mathrm{Al}$ año del diagnóstico, bajo anestesia general y a través de una incisión transversa de $4 \mathrm{~cm}$ sobre el arco costal afectado, extirpamos el $7^{\circ}, 8^{\circ}$ y $9^{\circ}$ cartílagos costales, preservando el pericondrio.

Transcurrido un año, el resultado ha sido satisfactorio.

\section{Caso 2}

Niña de 12 años, en seguimiento por la Unidad de enfermedades infecciosas pediátricas por un cuadro de dolor torácico izquierdo asociado a neumonía. Tras la resolución del cuadro neumónico el dolor torácico persistió, por lo que es derivada a la consulta de Neumología pediátrica, Ortopedia pediátrica y por último a la consulta de Cirugía pediátrica.

Presenta un cuadro de dolor costal izquierdo selectivo de 3 meses de evolución, que no se relaciona con cambios posturales ni movimientos respiratorios, es continuo y no se calma con analgésicos orales.

A la exploración, presenta un dolor más intenso a la palpación del borde costal izquierdo, localizando el punto más doloroso en la unión de la $10^{\mathrm{a}}$ costilla izquierda con la $11^{\mathrm{a}}$. Al realizar la maniobra del gancho, se reproduce el dolor. El resto de la exploración no revela signos patológicos, como tampoco la radiografía de tórax.

Se recomendó tratamiento antiinflamatorio inicial, descartando así otras posibilidades diagnósticas. Al no haber mejoría, se indicó la extirpación bajo anestesia general de la unión de la $10^{\underline{a}}$ costilla izquierda con la $11^{\mathrm{a}}$. No hubo complicaciones en el postoperatorio inmediato.

En ambos casos se apreció una resolución de los síntomas desde el primer día de postoperatorio. Tras cuatro años de evolución, podemos calificar el resultado como satisfactorio.

\section{DISCUSIÓN}

El SCD ha sido publicado en forma de casos clínicos aislados o pequeñas series $^{1-5}$. Probablemente sea una enfermedad infradiagnosticada ${ }^{2-7}$ y más aun en la población pediátrica. Hoy en día su etiología sigue siendo incierta pero se postula como causa más probable una movilidad excesiva de los cartílagos costales inferiores por inadecuada fijación ligamentosa, produciendo así una subluxación de los mismos que comprime los nervios intercostales contiguos $^{2,4}$.

Su diagnóstico es de exclusión ${ }^{1,5,8}$, basándose en un examen físico característico que consiste en la palpación de los cartílagos costales inferiores y la maniobra del gancho, que reproduce claramente la sintomatología ${ }^{2,3,6}$. En algún caso se ha utilizado la ecografía ${ }^{9}$ o la inyección de anestésico local intercostal ${ }^{2,3}$ como ayuda diagnóstica.

La sintomatología que produce es claramente incapacitante y al ser un dolor de origen neuropático, no calma con analgésicos ni antiinflamatorios habituales. Por ello se plantean soluciones agresivas pero eficaces, como la extirpación de los cartílagos costales dolorosos, conservando su pericondrio ${ }^{2,10}$. Esta intervención no está exenta de riesgos desde la producción de un neumotórax, deformidades torácicas residuales, recidiva del dolor o repercusión estética. En nuestra serie no hemos tenido complicaciones, consiguiendo resultados inmediatos y satisfactorios en ambos casos.

Existen autores que proponen medidas menos agresivas, como la inyección de toxina botulínica en los músculos intercostales $^{11} \mathrm{o}$ inyecciones periódicas con agentes anestésicos locales ${ }^{3}$ como tratamiento sintomático temporal. Estas medidas, aunque entrañan menos riesgos que la intervención radical, no han resultado tan eficaces como esta última.

Cabe destacar que aunque en estudios revisados ${ }^{2}$ recomienden la extirpación de 3 cartílagos, en nuestro último caso hemos encontrado una clara mejoría con la extirpación de un único cartílago costal. 
Quizás una cirugía de agresividad intermedia podría consistir en la unión mediante dos o tres gruesas suturas reabsorbibles de larga duración establecidas de forma percutánea, bajo control ecográfico, uniendo los últimos cartílagos costales. Bloquear el movimiento de fricción entre ambos podría ser resolutivo para el dolor, con una cicatriz imperceptible y un bajo riesgo quirúrgico. Esta actitud terapéutica mínimamente invasiva, con respaldo fisiopatológico, no descrita previamente en la literatura, sería nuestra sugerencia terapéutica para el SCD. De esta forma, reduciremos el impacto estético de dos maneras. Por un lado evitando la cicatriz cutánea y por otro la asimetría que produce la extirpación de la costilla en el contorno torácico, sin necesidad de aportar injerto cartilaginoso como se hace en otras patologías, así como cuando se extirpan costillas para reparar microtias $^{12,13}$.

Todos los estudios revisados en los que se opta por la cirugía ${ }^{2,3,10}$ tienen en común la ausencia de complicaciones, al igual que en los dos casos presentados y el carácter definitivo del procedimiento, sin recidivas o secuelas publicadas.

La cirugía de resección costal parece una opción muy agresiva, aunque con resultados muy satisfactorios. Un abordaje mínimamente invasivo podría ser mejor opción a considerar en el futuro.

\section{BIBLIOGRAFÍA}

1. TuRCIOs NL. Slipping rib syndrome in an adolescent: an elusive diagnosis. Clin Pediatr 2013; 52: 879-881.

2. Fu R, Iqbal CW, Jaroszewski DE, St Peter SD. Costal cartilage excision for the treatment of pediatric slipping rib syndrome. J Pediatr Surg 2012; 47: 1825-1827.
3. Saltzman DA, Schmitz ML, Smith SD, Wagner CW, JACKSON RJ, HARP S. The slipping rib syndrome in children. Paediatr Anaesth 2001; 11: 740743.

4. Mooney DP, SHorter NA. Slipping rib syndrome in childhood. J Pediatr Surg 1997; 32: 1081-1082.

5. TAubman B, Vetter VL. Slipping rib syndrome as a cause of chest pain in children. Clin Pediatr 1996; 35: 403-405.

6. Pérez Santos Jl, Tejón V, Llaneza J, Castaño A. En los niños con dolor abdominal... por favor, exploren las costillas. [Monografía en Internet] Web PEDiatrica. 2007. Fecha de consulta: 27/12/14. Disponible en: http://www. webpediatrica.com/casosped/pdf/79_costilla_deslizante.pdf

7. PORTER GE. Slipping rib syndrome: an infrequently recognized entity in children: a report of three cases and review of the literature. Pediatrics 1985; 76: 810-813.

8. Alsina Gibert M, Carnicer de la Pardina J, Ullot Font R, Escofet Soteras C, Muñoz GutiérRez JA. Síndrome de la costilla deslizante como causa de dolor abdominal recidivante en la edad pediátrica. An Esp Pediatr 1990; 32: 349-351.

9. Meuwly JY, Wicky S, Schnyder P, LePori D. Slipping rib syndrome: a place for sonography in the diagnosis of a frequently overlooked cause of abdominal or low thoracic pain. J Ultrasound Med 2002; 21: 339-343.

10. Copeland GP, Machin DG, Shennan JM. Surgical treatment of the slipping rib syndrome. Br J Surg 1984; 71: 522-523.

11. Pirali C, Santus G, Faletti S, De Grandis D. Botulinum toxin treatment for slipping rib syndrome: a case report. Clin J Pain 2013; 29: 1-3.

12. Kawanabe Y, Nagata S. A new method of costal cartilage harvest for total auricular reconstruction: Part I. Avoidance and prevention of intraoperative and postoperative complications and problems. Plast Reconstr Surg 2006; 117: 2011-2018.

13. Kawanabe Y, Nagata S. A new method of costal cartilage harvest for total auricular reconstruction: Part II. Evaluation and analysis of the regenerated costal cartilage. Plast Reconstr Surg 2007; 119: 308-315. 